\title{
ERROS DE PRESCRIÇÃO EM UMA FARMÁCIA BÁSICA DO DISTRITO FEDERAL
}

\author{
PRESCRIPTION ERRORS IN A BASIC PHARMACY \\ OF THE FEDERAL DISTRICT
}

\section{ERRORES DE PRESCRIPCIÓN EN UNA FARMACIA BÁSICA DEL DISTRITO FEDERAL}

\author{
Anna Cecília Soares Santos* \\ Cris Renata Grou Volpe** \\ Diana Lúcia Moura Pinho*** \\ Luciano Ramos De Lima**** \\ Marina Morato Stival ${ }^{* * * * *}$ \\ Valdiane Dutra Oliveira******
}

\begin{abstract}
RESUMO
Objetivo: Analisar prescrições eletrônicas e manuais quanto à ocorrência de polifarmácia e tipos potenciais de erros de medicação no contexto da atenção primária. Material e método: Estudo de caráter descritivo, transversal e retrospectivo, baseado na avaliação de prescriçóes manuais e eletrônicas arquivadas na farmácia da Unidade Básica de Saúde do Distrito Federal de Brasília (DF). Foram incluídas as segundas vias das prescriçóes para as doenças crônicas não transmissíveis. Resultados: 1500 prescriçōes foram analisadas. Apontaram a não totalidade dos critérios de avaliação proposto neste estudo, dentre eles: ilegibilidade (35,5\%), abreviaturas $(97,7 \%)$, forma farmacêutica $(57,6 \%)$, concentração (32,4\%). A polifarmácia foi identificada em $46 \%$ dos usuários e estava diretamente relacionada à idade do usuário. Conclusão: As prescriçóes apresentaram incompletude de informaçóes em relação ao que estabelece a legislação, são necessárias estratégias que busquem melhorar a promoção de saúde na atenção básica, como trabalhos de educação permanente e que visem sensibilizar os prescritores e demais atores envolvidos neste processo sobre o ato de prescrever medicamentos.
\end{abstract}

Palavras chave: Polifarmácia; Atenção primária; Saúde; Erros de prescrição; Enfermagen em Saúde Comunitária.

\footnotetext{
* Enfermeira. Docente da Estácio FATERN. Natal, Brasil. Email: anna_cecillia@hotmail.com

** Enfermeira. Docente da Universidade de Brasília. Brasília, Brasil. Email: crgrou@unb.br. Autor correspondente.

*** Enfermeira. Docente da Universidade de Brasília. Brasília, Brasil. Email: mourapinhodl@gmail.com

**** Enfermeiro. Docente da Universidade de Brasília. Brasília, Brasil. Email: ramosll@unb.br

***** Enfermeira. Docente da Universidade de Brasília. Brasília, Brasil. Email: marinamorato@unb.br

****** Enfermeira. Brasília, Brasil. Email: dutravaldiane@gmail.com
} 


\begin{abstract}
Objective: To analyze electronic and manual prescriptions regarding the occurrence of polypharmacy and potential types of medication errors in the context of primary care. Material and method: A descriptive, cross-sectional and retrospective study based on the evaluation of prescriptions filed at the pharmacy of a Basic Health Unit of the Federal District of Brazil. Copies of prescriptions for chronic non-communicable diseases were included. Results: 1500 prescriptions were analyzed according to evaluation criteria, such as illegibility (35.5 $\%$ ), abbreviations (97.7\%), dosage form (57.6\%), and concentration (32.4\%). Polypharmacy was identified in $46 \%$ of users and it was directly related to the age of the user. Conclusion: Prescriptions presented incomplete information regarding current legislation. Strategies that seek to improve health promotion in primary care, such as permanent education and more awareness from prescribers and other actors involved in the process of prescribing medications, are required.
\end{abstract}

Key words: Polypharmacy; Primary Health Care; Prescription erros; Enfermería en Salud Comunitaria.

\title{
RESUMEN
}

Objetivo: Analizar prescripciones electrónicas y manuales en cuanto a la ocurrencia de polifarmacia y tipos potenciales de errores de medicación en el contexto de la atención primaria. Material y método: Estudio de carácter descriptivo, transversal y retrospectivo, basado en la evaluación de prescripciones manuales y electrónicas archivadas en la farmacia de la Unidad Básica de Salud (UBS) del Distrito Federal (Brasil). Se incluyeron los duplicados de las prescripciones para las enfermedades crónicas no transmisibles. Resultados: Se analizaron 1.500 prescripciones considerando la no totalidad de los criterios de evaluación propuestos en este estudio, entre ellos: ilegibilidad (35,5\%), abreviaturas (97,7\%), forma farmacéutica $(57,6 \%)$, concentración $(32,4 \%)$. Se identificó polifarmacia en el $46 \%$ de los usuarios y que estaba directamente relacionada a la edad del usuario. Conclusión: Las prescripciones presentaron información incompleta en relación a lo establecido por la legislación. Se requieren estrategias que busquen mejorar la promoción de salud en la atención básica, como educación permanente y sensibilización de los prescriptores y demás actores involucrados en el acto de prescribir medicamentos.

Palabras clave: Polifarmacia; Atención Primaria a la Salud; Errores de prescripción; Community Health Nursing.

Fecha recepción: 31/05/2018 Fecha aceptación: 09/10/2018

\section{INTRODUÇÁO}

A segurança do paciente é parte inerente da prestação de assistência à saúde ${ }^{(1)}$ e deve estar presente em todas as etapas do cuidado especialmente durante o processo de medicação do paciente que abrange desde a prescrição até o monitoramento da adequada atuação da medicação no organismo do indivíduo, garantindo inclusive ausência de interaçóes medicamentosas ${ }^{(2-4)}$.

Dentro da perspectiva da segurança durante a medicação, erros de prescrição e a polifarmácia mais usualmente definida como o uso de cinco ou mais medicamentos ou ainda como o uso de 
mais medicamentos do que o necessário ${ }^{(5,6)}$ tem requerido atenção especial devido ao aumento de sua ocorrência. Estudos vêm evidenciando o aumento da associação da polifarmácia à eventos negativos para a saúde dos pacientes, sobretudo devido ao envelhecimento da população frequentemente associado as doenças crônicas não transmissíveis (DCNT) ${ }^{(5,7)}$.

O envelhecimento populacional que vem ocorrendo mundialmente nas últimas décadas ocasionado pela melhoria na qualidade e acesso a tecnologias da saúde tem acarretado em não somente mudança da pirâmide etária e no padrão de incidência e prevalência de doenças, mas também promove um aumento significativo no uso de medicamentos ${ }^{(8)}$, especialmente em pessoas idosas devido a taxa elevada de presença de multimorbidades que gira em torno de 50 a $98 \%$ e que requerem o uso de terapia medicamentosa ${ }^{(9)}$. $\mathrm{O}$ uso combinado de fármacos pode acarretar em diversos riscos em potencial, entre eles as interações medicamentosas, erros de administração e de prescrição inadequada, uso indevido de medicamentos, síndromes geriátricas e não adesão ao tratamento, além de poder resultar em diversos eventos adversos ${ }^{(5,10)}$.

Estratégias para aumentar a segurança durante a medicação, bem como diminuir a polimedicação e evitar erros de prescrição medicamentosa vem sendo amplamente discutidas em todos os níveis de saúde ${ }^{(10-12)}$. Na Atenção Primária em Saúde (APS), com vistas a garantir a prescrição segura de medicamentos e a qualidade da assistência prestada, novos estudos têm sido produzidos, assim como novas estratégias a fim de evitar a polimedicação também têm sido adotadas ${ }^{(12,13)}$.

Vale ressaltar que os erros de medicação estão entre os eventos adversos mais frequentes nesse nível de atenção e, em sua maioria, poderiam ter sido evitados em uma das fases do processo de medicação (prescrição, dispensação e administração) $)^{(14)}$. Dessa forma, é importante que, no processo de cuidado em saúde, os profissionais assegurem a qualidade da farmacoterapia evitandose o uso exacerbado de múltiplos fármacos, erros no processo de medicação, prescrição de medicamentos inapropriados e interações medicamentosas ${ }^{(15,16)}$.

Erro de medicação é atualmente um problema mundial de saúde pública, sendo o mais sério o de prescrição ${ }^{(6,17)}$. A prescrição de medicamentos é uma atividade importante para o processo de cuidados assistenciais aos pacientes ${ }^{(18)}$, e é vista como uma forma comum de atuação e relacionamento com o paciente. No entanto, cada decisão tomada com relação à prescrição, acarreta riscos que podem surgir em diversas nuances, desde a própria natureza do produto prescrito até sua forma de utilização e perfil do paciente assistido ${ }^{(19)}$.

Um estudo ${ }^{(18)}$ aponta que as prescrições têm papel ímpar na prevenção de erros de medicação e, sabe-se que tais erros podem decorrer de prescrições ambíguas, ilegíveis ou incompletas, ocasionando sérios danos ao paciente.

A legislação brasileira ${ }^{(20,21)}$ estabelece algumas normas para as prescriçóes a fim de assegurar sua qualidade, as quais necessitam apresentarse de forma clara, legível e de fácil compreensão, sem rasuras e abreviaturas. Cada prescrição deve apresentar informações relativas ao usuário do medicamento, ao medicamento e ao prescritor, além da data de elaboração do documento ${ }^{(20,21)}$. Conforme a legislação vigente, para que seja elaborada uma prescrição de forma admissível é necessário que essa esteja legível e tenha os principais componentes: nome do paciente, forma farmacêutica e a nomenclatura do medicamento, de acordo com Denominação Comum Brasileira (DCB), e na sua omissão, utiliza-se a Denominação Comum Inter-nacional (DCI). Informaçóes quanto ao uso interno ou externo dos medicamentos, via de administração, intervalos entre as doses, doses máxima por dia e duração do tratamento são essenciais para administração do medicamento. São obrigatórios a data explicitada, a assinatura e o carimbo do prescritor ${ }^{(20)}$.

A segurança na medicação é um dos requisitos para a segurança do paciente e manutenção da qualidade dos serviços em saúde conforme estabelecido pelo Programa Nacional de Segurança do Paciente (PNSP) e as respectivas responsabilidades das equipes de saúde e gestores com vistas a evitar danos aos pacientes ${ }^{(22)}$.

Frente ao exposto, o estudo teve como objetivo analisar as prescrições (eletrônicas e manuais) de usuários com hipertensão, diabetes, dislipidemias e doença cardiovascular, quanto à ocorrência da polifarmácia e dos tipos potenciais de erros de medicação, no contexto da Atenção Primária em uma Unidade Básica de Saúde (UBS), do Distrito Federal. 


\section{MATERIAL E MÉTODO}

Estudo descritivo, transversal e retros-pectivo de natureza quantitativa, baseado na avaliação de prescriçóes manuais e eletrônicas (segunda via), arquivadas na farmácia da UBS da Regiâo de Saúde Oeste de Brasília (DF). A coleta ocorreu entre 18 de julho a 29 de setembro de 2017.

Foram incluídas no estudo as segundas vias das prescrições, que possuíssem pelo menos um medicamento prescrito para as doenças crônicas não transmissíveis (DCNT) - Hipertensão Arterial Sistólica (HAS), Dia-betes Mellitus (DM), doença cardiovascular (DAC) e dislipidemia (DLP), de usuários de ambos os sexos e idades, referentes aos meses de janeiro a junho de 2017; e excluídas prescriçóes danificadas (rasgadas, apagadas ou ilegíveis), que dificultasse a identificação correta das informaçóes ou que não incluíam medicamentos para tratar as doenças citadas nas linhas anteriores.

O estudo analisou as variáveis, através de um instrumento de coleta de dados elaborado com base na legislação vigente no Brasil $^{(20,21)}$. Para realizar a verificação, os seguintes itens da prescrição foram analisados, com respostas do tipo sim ou não: legibilidade; usuários (nome completo, idade, gênero); prescritor (data; identificação do prescritor; categoria profissional); informaçôes sobre o medicamento (nome do medicamento legível, forma farmacêutica, posologia completa, dose, concentração, via de administração, nome comercial e princípio ativo); e a presença de itens vedados, que podem induzir a erros (rasuras ou emendas e escrita abreviada). As prescriçóes de medicamentos ainda foram classificadas de acordo com o tipo de redação, em manuais: elaboradas manualmente pelo prescritor e em eletrônicas: digitadas pelo prescritor por meio de um sistema computadorizado. Verificouse também a quantidade de medicamentos por prescrição e DCNT mais frequentes. A polifarmácia foi caracterizada como o uso de cinco ou mais medicamentos $^{(23)}$.

Para facilitar a coleta dos dados, os mesmos foram agrupados e organizados em planilhas do Microsoft Office Excel e para examinar as diferenças entre os grupos utilizaram-se análises descritivas e inferenciais. A análise foi realizada com auxílio do software Package for the Social Sciences $\left(\mathrm{SPSS}^{\oplus}\right)$ versão 18.0. As variáveis categóricas foram relatados como frequências absolutas e relativas, as variáveis numéricas foram relatados como média e desvio padrão (mínimo e máximo). As taxas de erros foram calculadas com base nas oportunidades de erros observadas. Todas as variáveis foram analisadas na univariável e multivariável. O nível de significância considerado foi de 5\%. O Odds Ratio (OR) foi calculado com intervalos de confiança (IC) de 95\%. Para associaçóes foram utilizados: Teste do qui-quadrado e Teste de Man-Whitney. Nesse estudo considerou os valores de $\mathrm{p}<0,005$ significativo.

O estudo obedeceu a Resolução do Conselho Nacional de Saúde $n^{\circ} 466 / 2012$. O projeto foi aprovado pelo Comitê de Ética em Pesquisa da Secretaria do Estado de Saúde do Distrito Federal (SES/DF) número 1.355.211.

\section{RESULTADOS}

Das 1500 prescriçóes analisadas, 979 (65\%) eram de usuários do sexo feminino e 521 (35\%) do sexo masculino. Dentre estes usuários, 691 (46\%) foram identificados com o uso de cinco ou mais medicaçóes, ou seja, a polifarmácia.

A maioria 462 (66,9\%) dos usuários em polifarmácia era do sexo feminino e esse estudo não apresentou significância quanto a este fator. Em contrapartida, ao analisar a idade e sua relação com a polifarmácia, observa-se que $51 \%$ dos usuários em polifarmácia tinham mais do que 50 anos de idade, além disso, usuários entre 61 a 70 anos eram os que mais tinham registro de polifarmácia em suas prescriçóes, correspondendo a $20,4 \%$ do total. Não obstante, ao contemplar a Tabela 1 e avaliar o valor de Odss Ratio é possível notar que quanto maior a idade maior é o risco de polifarmacia (71 anos, OR: 3,61 e IC 95\%: 1,99-6,55), sendo, portanto, o aumento da idade positivamente associado ao aumento da polimedicação. Todos os dados concernentes à idade dos usuários para quem foram prescritos os medicamentos apresentaram significância estatística.

Quanto à prevalência das doenças crônicas, a DM esteve presente em 453 (65,6\%) usuários, a HAS representou 659 (95,4\%), já 205 (29,7\%) usuários apresentaram DAC e $439(63,5 \%)$ a DLP. A presença de todas as DCNT apresentou significante e estiveram positivamente associados à polifarmácia, dentre as doenças crônicas as que mais apresentaram risco para a presença de polifarmácia foram: HAS 
erros de prescrição eM UMA FARMÁcia básica do.../ Soares A, Grou C, Moura D, Ramos L, Morato M, Dutra V.

podendo aumentando o risco de polimedicação em 5,47 vezes e a DLP aumentando o risco de presença de polifarmácia em 6,40 vezes.

Quanto ao tipo de redação, um total de 1.063 $(70,8 \%)$ prescriçōes foi realizado manualmente e $437(29,8 \%)$ eram prescriçōes eletrônicas. Dentre as prescriçōes eletrônicas, $429 \quad(98,2 \%)$ eram perfeitamente legíveis e compreensíveis, $3(0,7 \%)$ apresentavam impressão legível apenas em parte de seu escopo, enquanto $5(1,1 \%)$ não eram legíveis. No que se tangem as manuais, das 1.063 prescrições analisadas, apenas $686(64,5 \%)$ apresentavam grafia completamente legíveis, enquanto $24(2,3 \%)$ prescrições não possuíam grafia legível e 353 $(33,2 \%)$ prescriçóes apresentavam algum trecho na qual a leitura se fazia irrealizável (Tabela 2).

As rasuras estiveram presentes em $9(2,1 \%)$ prescriçōes eletrônicas e em 31 (2,9\%) prescriçôes manuais. Todas as prescrições, manuais e eletrônicas, expunham o nome do usuário a quem pertencia, em contrapartida, 11 (2,5\%) prescriçóes eletrônicas e 7 $(0,7 \%)$ prescriçóes manuais náo continham a data

Tabela 1. Características intrínsecas dos pacientes relacionados à polifarmácia na atenção primária. Ceilândia/Brasília, DF, Brasil, 2018.

\begin{tabular}{|c|c|c|c|c|c|c|c|c|}
\hline & & \multicolumn{4}{|c|}{ Polifarmácia } & \multirow[t]{3}{*}{$\mathbf{p}$} & \multirow[t]{3}{*}{ OR } & \multirow[t]{3}{*}{ IC 95\% } \\
\hline & & \multicolumn{2}{|c|}{$\operatorname{Sim}(691)$} & \multicolumn{2}{|c|}{ Não (809) } & & & \\
\hline & & $\mathbf{N}$ & $\%$ & $\mathbf{N}$ & $\%$ & & & \\
\hline \multirow[t]{2}{*}{ Sexo } & Feminino & 462 & 66,9 & 517 & 63,9 & 0,126 & 1,13 & $0,92-1,41$ \\
\hline & Masculino & 229 & 33,1 & 292 & 36,1 & & $\ldots$ & . - \\
\hline \multirow[t]{8}{*}{ Idade } & $<=20$ anos & 63 & 9,1 & 85 & 10,5 & $<0,000$ & 2,34 & $1,26-4,37$ \\
\hline & 21 a 30 anos & 58 & 8,4 & 74 & 9,1 & & 2,48 & $1,31-4,66$ \\
\hline & 31 a 40 anos & 97 & 14,0 & 120 & 14,8 & & 2,55 & $1,41-4,63$ \\
\hline & 41 a 50 anos & 96 & 13,9 & 124 & 15,3 & & 2,45 & $1,35-4,43$ \\
\hline & 51 a 60 anos & 105 & 15,2 & 123 & 15,2 & & 2,70 & $1,49-4,87$ \\
\hline & 61 a 70 anos & 141 & 20,4 & 127 & 15,7 & & 3,51 & $1,96-6,28$ \\
\hline & $>=71$ anos & 113 & 16,4 & 99 & 12,2 & & 3,61 & $1,99-6,55$ \\
\hline & SI & 18 & 2,6 & 57 & 7,0 & & $\ldots$ & $\ldots$ \\
\hline \multirow[t]{2}{*}{ DM } & Sim & 453 & 65,6 & 338 & 41,8 & $<0,000$ & 2,65 & $2,15-3,27$ \\
\hline & Não & 238 & 34,4 & 471 & 58,2 & & - - & - - \\
\hline \multirow[t]{2}{*}{ HAS } & Sim & 659 & 95,4 & 639 & 79,0 & $<0,000$ & 5,47 & $3,69-8,11$ \\
\hline & Não & 32 & 4,6 & 170 & 21,0 & & $\ldots$ & $\ldots$ \\
\hline \multirow[t]{2}{*}{ DAC } & Sim & 205 & 29,7 & 79 & 9,8 & $<0,000$ & 3,89 & $2,93-5,17$ \\
\hline & Não & 486 & 70,3 & 730 & 90,2 & & & - \\
\hline \multirow[t]{2}{*}{ DLP } & Sim & 439 & 63,5 & 173 & 21,4 & $<0,000$ & 6,40 & $5,09-8,04$ \\
\hline & Não & 252 & 36,5 & 636 & 78,6 & & - - & - - \\
\hline
\end{tabular}

*SI sem informação por se tratar de prescrições de paciente que não tinham registro no track.

em que foram realizadas. A ausência da data nas prescriçōes foi significativamente estatística e esta relacionada ao aumento de 1,83 vezes no risco de erro de prescrição medicamentosa.

Quanto ao nome do profissional prescritor, pôdese identificar que a maioria das prescriçóes estava com essa identificação, apenas $5(1,1 \%)$ prescrições eletrônicas e $6(0,6 \%)$ das manuais não exibiam esse dado. Já no que se diz respeito à classe profissional do prescritor, em todas as prescriçóes eletrônicas o responsável era o profissional médico, enquanto nas prescrições manuais somente 7 (0,7\%) foram realizadas por profissional enfermeiro. A identificação de registro de alergias do paciente foi identificada em apenas uma de cada tipo de prescrições, não apresentando significância estatística.

Quanto à presença do nome legível do medicamento, é essencial para a segurança de todo o processo de medicação do paciente, pois possibilita a adequada identificação do medicamento prescrito, 433 (99,1\%) das prescriçóes eletrônicas e 715 (67,3\%) das manuais, apresentavam o nome totalmente legível. Entre as prescrições eletrônicas, 216 (49,4\%) apresentavam a forma farmacêutica, já entre as prescriçóes manuais, sua maioria 612 (57,6\%) não continham. Pode-se notar que apesar de não apresentar significância estatística a ausência dessa 
Tabela 2. Distribuição de erros de medicação segundo tipo de prescrições (manuais e eletrônicas). Ceilândia/Brasília, DF, Brasil, 2018.

\begin{tabular}{|c|c|c|c|c|c|c|c|c|}
\hline & \multicolumn{4}{|c|}{ Tipo de prescriçáo } & \multirow[t]{3}{*}{$\mathbf{p}$} & \multirow[t]{3}{*}{ OR } & \multirow[t]{3}{*}{ IC 95\% } \\
\hline & & \multicolumn{2}{|c|}{ N Eletrônica } & \multicolumn{2}{|c|}{ N Manuais } & & & \\
\hline & & $\mathbf{N}$ & $\%$ & $\mathbf{N}$ & $\%$ & & & \\
\hline \multirow[t]{3}{*}{ Impressao é perfeitamente legível } & Sim & 429 & 98,2 & $\ldots$ & $\ldots$ & & & \\
\hline & Não & 5 & 1,1 & $\ldots$ & $\ldots$ & & & \\
\hline & Em parte & 3 & 0,7 & $\ldots$ & $\ldots$ & & & \\
\hline \multirow[t]{3}{*}{ Grafia é perfeitamente legível } & Sim & $-\cdots$ & $-\cdots$ & 686 & 64,5 & & & \\
\hline & Não & $-\ldots$ & $\ldots$ & 24 & 2,3 & & & \\
\hline & Em parte & $-\cdots$ & $-\cdots$ & 353 & 33,2 & & & \\
\hline \multirow[t]{2}{*}{ Rasuras na prescriçōes } & Sim & 9 & 2,1 & 31 & 2,9 & 0,349 & 1,09 & $0,92-1,30$ \\
\hline & Não & 428 & 97,9 & 1032 & 97,1 & & & \\
\hline \multirow[t]{2}{*}{ Nome do paciente } & Sim & 437 & 100,0 & 1063 & 100,0 & & & \\
\hline & Não & 0 & 0,0 & 0 & 0,0 & & & \\
\hline \multirow[t]{2}{*}{ Data } & Sim & 426 & 97,5 & 1056 & 99,3 & 0,003 & 1,83 & $1,02-3,27$ \\
\hline & Não & 11 & 2,5 & 7 & 0,7 & & & \\
\hline \multirow[t]{2}{*}{ Nome do prescritor } & Sim & 432 & 98,9 & 1057 & 99,4 & 0,232 & 1,30 & $0,75-2,23$ \\
\hline & Não & 5 & 1,1 & 6 & 0,6 & & & \\
\hline \multirow[t]{2}{*}{ Registro de alergias } & Sim & 1 & 0,2 & 1 & 0,1 & 0,516 & 0,70 & $0,17-2,82$ \\
\hline & Não & 436 & 99,8 & 1062 & 99,9 & & & \\
\hline \multirow[t]{2}{*}{ Profissional } & Enfermeiro & 0 & 0,0 & 7 & 0,7 & & & \\
\hline & Médico & 437 & 100,0 & 1056 & 99,3 & & & \\
\hline
\end{tabular}

informação, que pode auxiliar na identificação da via de administração da medicação, está relacionada com o aumento de até 2,36 vezes no risco de erro de medicação.

O registro da dose da medicação foi identificado em todas as prescriçóes eletrônicas, ao mesmo tempo em que 1.058 prescrições manuais continham a dose da medicação. A via de administração esteve ausente nas $156(35,7 \%)$ e $408(38,4 \%)$ prescriçôes eletrônicas e manuais, respectivamente. Apenas uma $(0,2 \%)$ prescrição eletrônica e 4 (0,4\%) prescriçôes manuais não continham a posologia. Ademais, a concentração do medicamento prescrito este presente $310(70,9 \%)$ prescriçōes eletrônicas e em $686(64,5 \%)$ prescrições manuais. O uso de abreviaturas não foi identificado em apenas $10(2,3 \%)$ prescriçõos eletrônicas e 192 (18,1\%) prescriçóes manuais. A presença de abreviaturas pode acarretar no aumento de 6,64 vezes a possibilidade de ocorrência de erros durante o processo de medicação do paciente. Quanto à nomenclatura, a utilização da Denominação Comum Brasileira (DCB) foi observada em $280(64,1 \%)$ prescriçóes eletrônicas e entre 870 (81,8\%) manuais. O tipo de nome contido na prescrição pode ser crucial durante a medicação já que essa variável apresentou significância estatística (Tabela 3).

\section{DISCUSSÃO}

Neste estudo, a legibilidade das prescrições manuais foi responsável por prescriçôes incompletas, ilegíveis ou pouco legíveis, demostrando que o fator de risco ilegibilidade foi praticamente eliminado com a prescrição eletrônica. Estudo abordando prescrições de medicamentos constatou que $47 \%$ das prescriçôes escritas à mão geraram erros no nome do paciente, em 33,7\% houve dificuldade na identificação do prescritor e 19,3\% estavam pouco legíveis ou ilegíveis. Segundo os autores quando comparadas, as prescriçôes manuais e informatizadas, estes índices podem diminuir, evidenciando uma redução de erros com o uso da prescrição eletrônica ${ }^{(6,24)}$.

O nome do usuário foi à única informação que esteve totalmente presente entre as prescriçóes. Dados semelhantes foram observados em uma farmácia do Município de Lajeado/RS $(96,6 \%)^{(25)}$. A prescrição faz parte do rol de documentos de ordem pessoal e intrasferível, portanto é necessário que contenha informaçóes especificas sobre o usuário para a qual foi dispensada. A falta de identificação pode acarretar no comprometimento do contato do farmacêutico após a dispensação em caso de necessidade ${ }^{(22)}$. Informações sobre a 
erros de prescrição em uma farmácia básica do.../ Soares A, Grou C, Moura D, Ramos L, Morato M, Dutra V.

identificação do prescritor foram observadas na maioria das prescriçôes, corroborando com achado de estudo realizado em uma farmácia comunitária da Paraíba, onde 99,3\% das prescriçóes continham assinatura do profissional; enquanto no Rio Grande do Sul esse dado mostrou-se presente, porém, um pouco menor $(83,2 \%)^{(25,26)}$. A identificação do prescritor é importante em função das responsabilidades legais.

Dentre as prescrições avaliadas, a maior parte era proveniente de médicos, seguido respectivamente de enfermeiros. Frequentemente, em UBS os profissionais enfermeiros fazem prescrição ou transcrição da prescrição médica durante as consultas de enfermagem, conforme estabelece a Lei $7.498 / 86^{(27)}$. Uma informação

Tabela 3. Distribuição de erros de medicação segundo tipo de prescrições (manuais e eletrônicas). Ceilândia/ Brasília, DF, Brasil, 2018.

\begin{tabular}{|c|c|c|c|c|c|c|c|c|}
\hline & & \multicolumn{4}{|c|}{ Tipo de prescrição } & \multirow[t]{3}{*}{$\mathbf{p}$} & \multirow[t]{3}{*}{ OR } & \multirow[t]{3}{*}{ IC 95\% } \\
\hline & & \multicolumn{2}{|c|}{$\begin{array}{c}\text { Eletrônicas } \\
\quad(437)\end{array}$} & \multicolumn{2}{|c|}{$\begin{array}{c}\text { Manuais } \\
(1063)\end{array}$} & & & \\
\hline & & $\mathbf{N}$ & $\%$ & $\mathbf{N}$ & $\%$ & & & \\
\hline \multirow[t]{3}{*}{ Nome do medicamento legível } & Sim & 433 & 99,1 & 715 & 67,3 & $<0,000$ & 0,06 & $0,00-0,48$ \\
\hline & Não & 1 & 0,2 & 25 & 2,4 & & $\ldots$ & $\ldots$ \\
\hline & parte & 3 & 0,7 & 323 & 30,4 & & 0,01 & $0,00-0,04$ \\
\hline \multirow[t]{3}{*}{ Forma farmacêutica } & Sim & 216 & 49,4 & 445 & 41,9 & 0,010 & 1,75 & $0,47-5,94$ \\
\hline & Não & 216 & 49,4 & 612 & 57,6 & & 2,36 & $1,21-7,81$ \\
\hline & parte & 5 & 1,1 & 6 & 0,6 & & $\ldots$ & $\ldots$ \\
\hline \multirow[t]{2}{*}{ Dose } & Sim & 437 & 100,0 & 1058 & 99,5 & 0,151 & 0,70 & $0,68-0,73$ \\
\hline & Não & 0 & 0,0 & 5 & 0,5 & & & \\
\hline \multirow[t]{2}{*}{ Via } & Sim & 281 & 64,3 & 655 & 61,6 & 0,348 & 1,08 & $0,92-1,28$ \\
\hline & Não & 156 & 35,7 & 408 & 38,4 & & & \\
\hline \multirow[t]{2}{*}{ Posologia } & Sim & 436 & 99,8 & 1059 & 99,6 & 0,653 & 1,45 & $0,25-8,4$ \\
\hline & Não & 1 & 0,2 & 4 & 0,4 & & & \\
\hline \multirow[t]{3}{*}{ Concentração } & Sim & 310 & 70,9 & 686 & 64,5 & 0,023 & 1,35 & $1,08-2,83$ \\
\hline & Não & 116 & 26,5 & 344 & 32,4 & & 1,01 & $0,98-2,01$ \\
\hline & parte & 11 & 2,5 & 33 & 3,1 & & $\ldots$ & $\ldots$ \\
\hline \multirow[t]{2}{*}{ Abreviaturas na prescrição } & Sim & 427 & 97,7 & 871 & 81,9 & $<0,000$ & 6,64 & $3,61-12,22$ \\
\hline & Não & 10 & 2,3 & 192 & 18,1 & & & \\
\hline \multirow[t]{3}{*}{ Tipo de nome } & nercial & 12 & 2,7 & 16 & 1,5 & $<0,000$ & $\ldots$ & - - \\
\hline & o ativo & 280 & 64,1 & 870 & 81,8 & & 2,33 & $1,08-4,98$ \\
\hline & Ambos & 145 & 33,2 & 177 & 16,7 & & 0,91 & $0,41-199$ \\
\hline
\end{tabular}

importante e que na maioria das vezes passa despercebida é a data, esta deve ser indispensável e relevante para assegurar a validade da prescrição, pois indica a continuidade de um tratamento ou reutilização de uma prescrição. Nesse estudo, a ausência dessa informação esteve relacionada ao aumento do risco de erros de medicação.

Os erros encontrados com maior frequência em nosso estudo foram ausência de forma farmacêutica, via de administração e concentração, em ambas as prescrições. Estudos apontam que a falta de informaçóes essenciais sobre o medicamento, tais como: concentração, forma farmacêutica, via de administração e posologia (dose, frequência e duração do tratamento), pode levar ao desperdício de recursos financeiros, além de prejuízo terapêutico ao usuário ${ }^{(28)}$. A dose incorreta dos medicamentos foi o tipo de erro mais frequente encontrado em uma Atenção Primária da Suíça. Os incidentes identificados nesse estudo estavam, em sua maioria, relacionados à pacientes portadores de doenças crônicas, idosos em uso de polifarmácia e com ausência de orientação do profissional ao paciente ${ }^{(29)}$.

Isso implica em tratamentos inadequados e ineficazes, podendo refletir uma possível diminuição da qualidade do atendimento realizado pelo prescritor, em uma dispensação errônea, pelo dispensador ou em um tratamento de difícil adesão para o usuário, devido à ausência de 
informações necessárias para sucesso na terapêutica medicamentosa ${ }^{(30)}$.

A despeito da alta prevalência de prescrição pela denominação genérica encontrada neste estudo, o valor encontrado não é o ideal já que o recomendado é que $100 \%$ dos medicamentos sejam prescritos em consonância com a nomenclatura da $\mathrm{DCB}^{(25)}$. Cabe ressaltar que o hábito de prescrever pelo nome genérico pode ter grande impacto na racionalização das ações da assistência farmacêutica, reduzindo os custos de aquisição desses produtos e facilitando a adoção de protocolos ou padronização de esquemas terapêuticos. Além disso, a utilização do nome comercial se dá pelo marketing e por pressão da indústria farmacêutica sobre os profissionais prescritores ${ }^{(25,31,32)}$.

A utilização de abreviaturas está entre as causas mais citadas de erros de medicação por seu potencial de confusão e falhas de comunicação. Neste estudo, a quase totalidade das prescrições apresentaram abreviaturas, apenas uma pequena porcentagem das prescriçóes analisadas não continham abreviaturas. As ações de prevenção de erros associados às abreviaturas são simples, efetivas e factíveis em qualquer instituição de saúde. Elas se fundamentam na educação dos profissionais sobre o tema e na elaboração e ampla divulgação de uma lista contendo as abreviaturas, siglas e símbolos que nunca devem ser empregados e os respectivos riscos associados ao seu uso ${ }^{(33)}$.

Frente a essa realidade, sugere-se que as unidades de saúde elaborem, formalizem e divulguem um manual ou lista de siglas e abreviaturas padronizadas em concordância com a literatura e terminologia técnica recomendadas, de uso obrigatório a serem utilizados no registro de documentos de todos os profissionais, de modo a promover e melhorar a comunicação entre os membros da equipe de saúde.

A rasura apesar de não ter sido frequente mostrou-se presente, assim como ausência de registro de alergias na maioria das prescriçóes. Destaca-se a importância da colaboração entre os prescritores para que se consiga atingir a padronização das prescriçôes, diálogos abertos entre a equipe interprofissional e pacientes, para diminuir as dúvidas relativas às prescrições ${ }^{(26)}$.

A hipertensão, corroborando com outros estudos $^{(11,34)}$, foi a condição crônica mais frequente e apresentou associação intensa com a polifarmácia.
O uso de medicamentos crônicos por idosos é uma importante dimensão a ser considerada na assistência ao idoso e a polifarmácia surge como um indicador para o uso mais seguro e eficaz dos medicamentos, evitando o risco de iatrogenia, alterações na farmacodinâmica e farmacocinética, efeitos adversos e pioras funcionais ${ }^{(34,35)}$.

A polifarmácia encontrada neste estudo mostrou-se semelhante à relatada por entrevistados em um município de São Paulo ${ }^{(34)}$. A média de medicamentos por prescrição verificada no presente estudo talvez possa ser justificada ao considerarmos a predominância das doenças crônicas, que leva os usuários a fazerem uso de múltiplas medicaçôes, e dessas doenças estarem associadas a outras comorbidades. Entre os fatores que contribuem para a polifarmácia, as doenças crônicas são as que apresentam maior relevância e associação ${ }^{(36)}$. A epidemia de DCNT resulta em consequências devastadoras para os indivíduos, famílias e comunidades, além de sobrecarregar os sistemas de saúde ${ }^{(11)}$.

Alguns fatores podem contribuir para o consumo elevado de medicamentos, como a baixa frequência de uso de tratamentos não farmacológicos para as doenças crônicas e/ou outros problemas de saúde e o fácil acesso as medicaçôes ${ }^{(36)}$. De modo que os usuários devem ser encorajados e estimulados, por meio das políticas públicas disponíveis, quanto à importância da aderência a medidas não medicamentosas no controle e redução das doenças crônicas, o que consequentemente reduziria o uso não racional e excessivo de medicamentos.

A faixa etária da população idosa observada no presente estudo foi semelhante à identificada em um estudo ${ }^{(25)}$ na UBS de Lajeado/RS e em uma farmácia comunitária de São Luiz do Gonzaga/ $\mathrm{RS}^{(37)}$. A idade é um fator com grande relevância, principalmente em relação às orientações para crianças e idosos, a fim de evitar superdosagem ou medicamentos inadequados a estas faixas etárias. Logo, garantir a segurança da farmacoterapia nos pacientes idosos é uma tarefa complexa. Devem-se considerar as alteraçóes fisiológicas e consequentes mudanças no perfil farmacocinético e farmacodinâmico de inúmeros fármacos, além da presença de um quadro de morbidades que tende a potencializar tais modificaçóes ${ }^{(38)}$.

É relevante destacar que nem sempre o uso 
de múltiplas medicaçóes pelos idosos ocorre de maneira equivocada, já que muitos necessitam dos medicamentos para realizar o controle de doenças e sinais e sintomas; porém taxas elevadas de emprego da polifarmácia estão mais frequentemente relacionadas ao aumento de uso indevido de medicações e às interações medicamentosas demandando a precaução dos profissionais de saúde a fim de evitar erros de medicação ${ }^{(39,40)}$. Portanto, o principal desafio para qualificar a atenção em saúde é garantir que a prescrição de múltiplos medicamentos seja apropriada e segura. Medidas como a revisão dos medicamentos e a potencial desprescrição devem ser avaliadas, principalmente por médicos generalistas ou farmacêuticos, a fim de personalizar o tratamento em pessoas com multimorbidade ou vulnerabilidade específica ${ }^{(11)}$.

O uso de múltiplos medicamentos, ou polifarmácia, é comum e crescente na prática clínica, principalmente em pessoas acima de 65 anos. Dentre as razóes que explicam esta prática, destacamse os tratamentos não baseados em evidências, a adoção de combinaçóes com potenciais interaçóes medicamentosas; o tratamento farmacológico dos efeitos secundários de outros medicamentos; e a prescrição simultânea, por vários médicos, sem que ocorra a necessária conciliação terapêutica para o paciente $^{(11)}$.

Apesar do sexo feminino não ter apresentado diferenças significativas quanto ao masculino, em outros estudos o sexo feminino esteve associado ao maior uso de polifarmácia ${ }^{(41,42)}$. Os motivos das razóes do uso de medicamentos entre idosas, independente da polifarmácia, parecem estar ligados a diversas variáveis, dentre elas as de ordem biológica, as de ordem psicológica e também as variáveis de ordem sociocultural ${ }^{(41)}$.

A atenção primária à saúde insegura propicia a diminuição da qualidade do cuidado prestado aos usuários. Logo, a identificação de potenciais ricos para erros é crucial para a identificação de possíveis soluçóes e para a difusão e melhora de medidas que fortalecem a segurança do paciente e o trabalho desenvolvido em equipe na $\operatorname{APS}^{(11)}$.

\section{CONCLUSÃO}

Os resultados mostraram que nenhuma das prescriçôes apresentava todos os requisitos legais exigidos, revelando déficits de informação necessários e obrigatórios. Pôde-se observar que as prescrições manuais apresentaram frequentes erros, além disso, a prescrição eletrônica mostrou redução desses erros em relação à prescrição manuscrita. Observa-se que a prescrição de medicamentos demanda sensibilizaçáo dos profissionais prescritores a fim de evitar potenciais erros de medicação, tais como os relatados nesta investigação.

Os resultados do estudo promovem a necessidade de avançar para a prescrição eletrônica para melhorar a qualidade da prescrição e a segurança do paciente. Além disso, o estudo também enfatiza a importância e responsabilização da escrita completa com prescrição clara e legível, por parte dos prescritores. Também destaca a necessidade de mais programas de treinamento, educação continuada e avaliação regular das habilidades de prescrição para minimizar o risco de erros de medicação e como isso imprimir segurança no ciclo do uso do medicamento e melhoria da qualidade das prescriçóes.

As limitações deste estudo incluíram a impossibilidade dos resultados serem estendidos a outros países já que a APS assume diferentes arranjos no contexto de saúde de cada país e a abrangência do estudo, realizado em apenas uma UBS, além disso, a falta de maiores informaçóes sobre os usuários de medicamentos impede mensurar detalhadamente quais os possíveis problemas que os erros de prescrição poderiam ocasionar, demonstrando negligencia de informaçóes por parte dos profissionais de saúde.

Sendo assim, como agenda futura de pesquisa, novos estudos com essa temática podem ser realizados abrangendo um maior quantitativo de participantes e UBS, assim como, a reconciliação e uso inapropriado de medicamentos para pessoas idosas, visando o desenvolvimento de políticas de segurança do paciente e reduçáo de erros de medicação, destaca-se também a necessidade de realização de estudos com o foco nas interações medicamentosas no contexto da Atenção Primária em saúde a fim de subsidiar protocolos de cuidado.

Espera-se que os resultados deste estudo possam contribuir para alertar e conscientizar os prescritores quanto à importância de uma prescrição de medicamentos segura, possibilitando assim a melhoria da qualidade na assistência e segurança no uso de medicamentos na atenção primária. 


\section{REFERENCIAS}

1. Pena MM, Braga AT, Meireles ES, Vassão LGC, Melleiro MM. Mapeamento dos erros de medicação em um hospital universitário. Revista Enfermagem Uerj [Internet]. 2016 Jun [citado 2018 Out 2]; 24(3):1-6. Disponível em: https://doi.org/10.12957/ reuerj.2016.7095

2. Mesquita KO, Silva LCC, Lira RCM, Freitas CASL, Lira GV. Segurança do Paciente na Atenção Primária à Saúde: Revisão Integrativa. Cogitare Enferm [Internet]. 2016 Abr-Jun [citado 2018 Out 2]; 21 (2): 1-8. Disponível em: https://revistas.ufpr. br/cogitare/article/view/45665/28526

3. Kim L, Lyder CH, McNeese-Smith D, Leach LS, Needleman J. Defining attributes of patient safety through a concept analysis. J Adv Nurs. 2015; 71(11): 2490-503.

4. Ulrich B, Kear T. Patient safety and patient safety culture: Foundations of excellent health care delivery. Neph Nur J. 2014; 41 (5): 447-56.

5. Maher RL, Hanlon J, Hajjar ER. Clinical consequences of polypharmacy in elderly. Expert Opin Drug Saf. 2013; 13(1): 57-65.

6. Albarrak AI, Al Rashidi EA, Fatani RK, AI Ageel SI, Mohammed R. Assessment of legibility and completeness of handwritten and electronic prescriptions. Saudi Pharm J. 2014; 22 (6): 522-27.

7. Kim HA, Shin JY, Kim MH, Park BJ. Prevalence and Predictors of Polypharmacy among Korean Elderly. PloS One [Internet]. 2014 Jun 10 [cited 2018 Out 2]; 9(6): e98043. Available from: https://www. ncbi.nlm.nih.gov/pmc/articles/PMC4051604/

8. Thio SL, Nam J, Van Driel ML, Dirven T, Blom JW. Effects of discontinuation of chronic medication in primary care: a systematic review of deprescribing trials. Br J Gen Pract. 2018; 68(675):663-72.

9. Cavalcanti G, Doring M, Portella MR, Bortoluzzi EC, Mascarelo A, Dellani MP. Multimorbidity associated with polypharmacy and negative selfperception of health. Rev. Bras. Geriatr. Gerontol. 2017; 20(5): 634-42.

10. Al Khaja KAJ, Ahmed Isa $H$, Veeramuthu $S$, Sequeira RP. Potentially Inappropriate Pres-cribing in Older Adults with Hypertension or Diabetes Mellitus and Hypertension at Primary Care Setting in Bahrain. Med Princ Pract. 2018; 27(3): 241-49.

11. Nascimento RCRM, Álvares J, Guerra AA Junior, Gomes IC, Silveira MR, Costa EA, et al. Polypharmacy: a challenge for the primary health care of the Brazilian Unified Health System. Rev Saude Publica [Internet]. 2017 Nov 13 [cited 2018 Out 2]; 51(suppl 2): 19s. Available from: https:// www.ncbi.nlm.nih.gov/pubmed/29160460

12. Herr GEG, Aozane F, Kolankiewicz ACB. Segurança do paciente: uma discussão necessária
Rev. Eletrônica Gestão \& Saúde [Internet]. 2015 Jun [citado 2018 Out 2]; 6(3): 2300-310. Disponível em:https://dialnet.unirioja.es/descarga/ articulo/5560284.pdf

13. Marques LFG, Romano-Lieber NS. Estratégias para a segurança do paciente no processo de uso de medicamentos após alta hospitalar. Physis [Internet]. 2014 Mai 2 [citado 2018 Out 2]; 24(2): 401-20. Disponível em: http://www.scielo.br/scielo. php?pid=S0103-73312014000200401\&script $=$ sci abstract $\&$ tlng $=p$

14. Agência Nacional de Vigilância Sanitária Brasil. Assistência segura: uma reflexão teórica aplicada à prática [Internet]. Brasília: ANVISA; 2017 [citado 2017 Jun 20]. 170 p. Disponível em:https:// www20.anvisa.gov.br/segurancadopaciente/index. $\mathrm{php/publicacoes/item/caderno-1-assistencia-}$ segura-uma-reflexao-teorica-aplicada-a-pratica

15. Marković-Peković V, Škrbić R, Petrović A, Vlahović-Palčevski V, Mrak J, Bennie M, et al. Polyphar-macy among the elderly in the Republic of Srpska: extent and implications for the future. Expert Rev Pharmacoecon Outcomes Res 2015; 16(5): 609-18.

16. Cadogan CA, Ryan C, Hughes CM. Appropriate polypharmacy and medicine safety: when many is not too many. Drug Saf. 2016; 39(2): 109-16.

17. ISMP-Brasil. Erros de medicaçáo asso-ciados a abreviaturas, siglas e símbolos. [Internet]. Belo Horizonte: ISMP [citado 2018 Jul 10]. Disponível em: http://www.ismp-brasil.org/site/wp-content/ uploads/2015/07/V4N2.pdf.

18. Bonadiman RL, Bonadiman RL, Bonadiman SL, Silva DA da. Estudo das prescriçôes medicamentosas em uma farmácia básica de Itapemirim, Espírito Santo - Brasil. Acta Biomedica Brasiliensia. 2013; 4(2): 114-23.

19. Avery AJ, Ghaleb M, Barber N, Dean Franklin B, Armstrong SJ, Serumaga B, et al. The prevalence and nature of prescribing and monitoring errors in English general practice: a retrospective case note review. Br J Gen Pract. 2013; 63(613): 543-53.

20. Brasil. Lei ${ }^{\circ}$ 5.991, de 17 de dezembro de 1973. Dispóe sobre o controle sanitário do comércio de drogas, medicamentos, insumos farmacêuticos e correlatos, e dá outras providências [Internet]. Diário Oficial da Uniâo, Brasília 19 dez 1973 [citado 2018 Out 2]. Disponível em: URL http:// www.cff.org.br/userfiles/file/leis/5991.pdf

21. Portaria N. 3.916 de 30 de outubro de 1998. Aprova a Política Nacional de Medicamentos [Internet] Diário Oficial da União, Brasília 1998 Out 30 [citado 2018 Out 2]. Disponível em: http://bvsms.saude.gov.br/bvs/saudelegis/gm/1998/ 
prt3916_30_10_1998.html

22. Salvador AS, Schelbauer SA, Lovatto MOS, Krieger D. Segura-nça do Paciente no Processo Medicamentoso. Revista UNIPLAC [Internet]. 2016 [citado 2018 Out 2]; 4 (1). Disponível em: https://revista.uniplac.net/ojs/index.php/uniplac/ article/view/2504

23. Secoli, SR. Polifarmácia: interaçôes e reaçóes adversas no uso de medicamentos por idosos. Rev Bras Enferm. 2010; 63(1): 136-40.

24. Volpe CRG, Melo EM, Aguiar LB, Pinho DL, Stival MM. Risk factors for medication errors in the electronic and manual prescription. Rev. Lat Am Enfermagem [Internet]. 2016 Aug 8 [cited 2018 Out 2]; 24: e2742. Available from: https:// www.ncbi.nlm.nih.gov/pubmed/27508913

25. Laste G, Silva Torres IL, Deitos A, Souza AC, Souza A, Kauffmann C, et al. Análise das prescriçóes médicas dispensadas em farmácia no sistema único de saúde. Revista HCPA. 2013; 33 (1):15-25.

26. Morais VD, Comarella L, Morais JD. Avaliação da qualidade das prescriçóes medicamentosas dispensadas em uma unidade de saúde da família no município de João Pessoa, Paraíba. Rev. Eletr. Farm [Internet]. 2018 [citado 2018 Out 2]; 14(4):17-27. Disponível em: https://www.revistas.ufg.br/REF/ article/view/44467

27. Lei n. 7.498, de 25 de junho de 1986: dispóe sobre a regulamentaçáo do exercício da enfermagem e dá outras providências [Internet]. Diário Oficial da União, Brasília 26 Jun 1986 [citado 2018 Out 2]. Disponível em: http://www.planalto.gov.br/ ccivil_03/LEIS/L7498.htm

28. Silva dos Santos D, Souza OV, Souza Nascimento AL, Pereira J, Costa Santos MJ, Costa Alves M, et al. Segurança do paciente: fatores causais de eventos adversos a medicamentos pela equipe de enfermagem. Ciências Biológicas e da Saúde. 2014; 2(2): 19-30.

29. Gnädinger M, Conen D, Herzig L, Puhan MA, Staehelin A, Zoller M, et al. Medication incidents in primary care medicine: a prospective study in the Swiss Sentinel Surveillance Network (Sentinella). BMJ Open [Internet]. 2017 Sept 1 [cited 2018 Out 2]; 7(7): e013658. Available from: https://bmjopen. bmj.com/content/7/7/e013658

30. Assiri GA, Shebl NA, Mahmoud MA, Aloudah $\mathrm{N}$, Grant E, Aljadhey $\mathrm{H}$, et al. What is the epidemiology of medication errors, error-related adverse events and risk factors for errors in adults managed in community care contexts? A systematic review of the international literature. Bmj Open [Internet]. 2018 May 5 [cited 2018 Out 2]; 8(5): e019101. Available from: https://www.ncbi.nlm. nih.gov/pubmed/29730617

31. Gimenes FRE, Marques TC, Teixeira TCA, Mota MLS, Silva AEBC, Cassiani SHB. Administração de medicamentos, em vias diferentes das prescritas, relacionada a prescrição médica. Rev Lat Am Enfermagem [Internet]. $2011 \mathrm{Jan}-\mathrm{Fev}$ [citado 2018 Out 2]; 19(1). Disponível em: http://www.scielo.br/ pdf/rlae/v19n1/pt_03.pdf

32. Zanin GD, Luz HS. Aspectos Legais de Prescrições Médicas Aviadas em uma Farmácia Comunitária do Município de Santa Tereza do Oeste, Paraná. Rev Thêm et Scientia [Internet]. 2012 Jan-Jun [citado 2018 Out 2]; 2(1):1-7. Disponível em:http://www. themaetscientia.com/index.php/RTES/article/ view/60/63

33. Instituto para Práticas Seguras no Uso de Medicamentos do Brasil (ISMP Brasil). Erros de medicação associados a abreviaturas, siglas e símbolos. [Internet]. ISMP Brasil; 2016. [citado 2018 Mai 11]. Disponível em: http://www.ismpbrasil.org/site/wp-content/uploads/2015/07/V4N2. pdf

34. Carvalho MFC, Romano-Lieber NS, BergstenMendes G, Secoli SR, Ribeiro E, Lebrão ML, et al. Polifarmácia entre idosos do Município de São Paulo - Estudo SABE. Rev Bras Epidemiol. 2012;15(4):817-27.

35. Abdulah R, Insani WN, Destiani DP, Rohmaniasari N, Mohenathas ND, Barliana MI, et al. Polypharmacy leads to increased prevalence of potentially inappropriate medi-cation in the Indonesian geriatric population visiting primary care facilities. Ther Clin Risk Manag [Internet]. 2018 Sept 4 [cited 2018 Out 2]; 14: 1591-597. Available from: https://www.dovepress.com/ polypharmacy-leads-to-increased-prevalence-ofpotentially-inappropriat-peer-reviewed-articleTCRM

36. Silveira EA, Dalastra L, Pagotto V. Polifarmácia, doenças crônicas e marcadores nutricionais em idosos. Rev Bras Epidemiol. 2014; 17(4): 818-29.

37. Silva ERB, Bandeira VAC, Oliveira KR. Avaliação das prescriçóes dispensadas em uma farmácia comunitária no município de São Luiz GonzagaRS. Rev Ciênc Farm Básica Apl. 2012; 33 (2): 275 81.

38. Instituto para Práticas Seguras no Uso de Medicamentos do Brasil (ISMP Brasil). Desafio global de segurança do paciente medicação sem danos [Internet]. ISMP Brasil; 2018 Fev [citado 2018 Out 2]. Disponível em: http://www.ismpbrasil.org/site/wp-content/uplo-ads/2018/02/ ISMP_Brasil_Desafio_Global.pdf .

39. Santos TRA, Lima DM, Nakatani AYK, Pereira 
LV, Leal GS, Amaral RG. Consumo de medicamentos por idosos, Goiânia, Brasil. Rev. Saúde Pública. 2013; 47(1): 94-103.

40. Hedna K, Hakkarainen KM, Gyllensten $\mathrm{H}$, Jönsson AK, Petzold M, Hägg S. Potentially inappropriate prescribing and adverse drug reactions in the elderly: a population-based study. Eur J Clin Pharmacol. 2015; 71(12):1525-33.

41. Herr M, Robine JM, Pinot J, Arvieu JJ, Ankri J.
Polypharmacy and frailty: prevalence, relationship, and impact on mortality in a French sample of 2350 old people. Pharmacoepidemiol Drug Saf. 2015; 24(6): 637-46.

42. Díez-Manglano J, Giménez-López M, GarcésHorna V, Sevil-Puras M, Castellar-Otín E, González-García P, et al. Excessive polypharmacy and survival in polypathological patients. Eur J Clin Pharmacol. 2015; 71(6): 733-39. 